\title{
A New Approximate Analytical Method for ODEs
}

\author{
HOSSEIN AMINIKHAH
}

\begin{abstract}
In this paper, we propose a new algorithm for solving ordinary differential equations. We show the superiority of this algorithm by applying the new method for some famous ODEs. Theoretical considerations are discussed. The first He's polynomials have used to reach the exact solution of these problems. This method which has good stability and accuracy properties is useful in deal with linear and nonlinear system of ordinary differential equations.
\end{abstract}

Mathematics Subject Classification 2000: 34A24, 34A45, 55P05

Additional Key Words and Phrases: perturbation technique, He's polynomials, ordinary differential equations

\section{INTRODUCTION}

Differential equations have wide applications in various engineering and science disciplines. In general, modeling of the variation of a physical quantity, such as temperature, pressure, displacement, velocity, stress, strain, current, voltage, or concentration of a pollutant, with the change of time or location, or both would result in differential equations. Similarly, studying the variation of some physical quantities on other physical quantities would also lead to ordinary differential equations. Many famous mathematicians have studied differential equations and contributed to the field, including Newton, Leibniz, the Bernoulli family, Riccati, Lane, Emden, Clairaut, Euler and Lagrange. The various powerful numerical and analytic methods such as perturbation methods, Nayfeh (2011), Rand et al. (1987), He (1999), Ganji (2006), Abbasbandy (2006), Yildirım et al. (2009), Shakeri et al. (2008), Aminikhah (2010) and Aminikhah et al. (2009), Adomian decomposition method, Bildik et al. (2006), Biazar et al. (2004), Babolian et al. (2004) and Pamuk (2005), homotopy analysis method, Liao (2004), Abbasbandy (2006) and Jafari et al. (2012) and differential transform method, Odibat et al. (2008), Ertürk et al. (2008) and Ayaz (2004) have been developed for solving them.

There are some methods to obtain approximate and analytical solutions of this kind of equations. The purpose of this article is to extend the new iterative method with a reliable algorithm for solving the general linear and nonlinear ordinary differential equations. This algorithm is based on perturbation technique and Laplace transform. Several examples, including some well known problems, will present to show the ability of the new method. 


\section{ANALYSIS OF THE METHOD}

In this section, we illustrate basic idea of the proposed approach. Consider the following nonlinear equation:

$$
L(u)+N(u)=f(t)
$$

with the following initial conditions

$$
u(0)=\alpha_{0}, u^{\prime}(0)=\alpha_{1}, \ldots, u^{(n-1)}(0)=\alpha_{n-1}
$$

where $L$ is a linear, $N$ is a nonlinear operator and $f(t)$ is a known analytical function.

By the homotopy technique, we construct a following equation

$$
L(v)-u_{0}+p\left\{N(v)+u_{0}-f(t)\right\}=0,
$$

where $p \in[0,1]$ is an embedding parameter, $u_{0}=\sum_{n=0}^{\infty} \beta_{n} Q_{n}(t)$ and $\beta_{0}, \beta_{1}, \beta_{2}, \ldots$ are unknown coefficients and $Q_{0}(t), Q_{1}(t), Q_{2}(t), \ldots$ are specific functions depending on the problem.

It is assumed that the unknown function $v(t)$ can be expressed by an infinite series, $v=\sum_{n=0}^{\infty} p^{n} v_{n}$, and nonlinear term $N(v)$ can be decomposed into an infinite series of polynomials given by

$$
N(v)=\sum_{n=0}^{\infty} H_{n}\left(v_{0}, v_{1}, \ldots, v_{n}\right)
$$

where $H_{n}\left(v_{0}, v_{1}, \ldots, v_{n}\right)$ are called He's polynomials and proposed by Ghorbani (2009), and are defined by

$$
H_{n}\left(v_{0}, v_{1}, \ldots, v_{n}\right)=\frac{1}{n !}\left[\frac{d^{n}}{d p^{n}} N\left(\sum_{k=0}^{n} p^{k} v_{k}\right)\right]_{p=0}, n=0,1,2, \ldots
$$

Obviously, when $p=1$, from (3) we have original equation (1).

Now let us write the equation (3) in the following form

$$
L(v)=\sum_{n=0}^{\infty} \beta_{n} Q_{n}(t)-p\left(\sum_{n=0}^{\infty} H_{n}\left(v_{0}, v_{1}, \ldots, v_{n}\right)+\sum_{n=0}^{\infty} \beta_{n} Q_{n}(t)-f(t)\right)
$$

where $L(v)=v^{(n)}$. 
By applying Laplace transform on both sides of (6), we have

$$
\begin{aligned}
& s^{n} \mathcal{L}\{v\}-s^{n-1} v(0)-s^{n-2} v^{\prime}(0)-\cdots-v^{(n-1)}(0)= \\
& \mathcal{L}\left\{\sum_{n=0}^{\infty} \beta_{n} Q_{n}(t)-p\left(\sum_{n=0}^{\infty} H_{n}\left(v_{0}, v_{1}, \ldots, v_{n}\right)+\sum_{n=0}^{\infty} \beta_{n} Q_{n}(t)-f(t)\right)\right\}
\end{aligned}
$$

or

$$
\begin{aligned}
& \sum_{n=0}^{\infty} p^{n} v_{n}=\mathcal{L}^{-1}\left\{\frac{1}{s^{n}}\left(s^{n-1} v(0)+s^{n-2} v^{\prime}(0)+\cdots+v^{(n-1)}(0)\right)\right. \\
& \left.+\frac{1}{s^{n}} \mathcal{L}\left\{\sum_{n=0}^{\infty} \beta_{n} Q_{n}(t)-p\left(\sum_{n=0}^{\infty} H_{n}\left(v_{0}, v_{1}, \ldots, v_{n}\right)+\sum_{n=0}^{\infty} \beta_{n} Q_{n}(t)-f(t)\right)\right\}\right\}
\end{aligned}
$$

Suppose that the initial approximation has the form $v(0)=\alpha_{0}, v^{\prime}(0)=\alpha_{1}, \ldots, v^{(n-1)}(0)=\alpha_{n-1}$ and comparing coefficients of terms with identical powers of $p$, leads to

$$
\begin{aligned}
& v_{0}(x)=\mathcal{L}^{-1}\left\{\frac{1}{s} \alpha_{0}+\frac{1}{s^{2}} \alpha_{1}+\cdots+\frac{1}{s^{n}} \alpha_{n-1}+\frac{1}{s^{n}} \sum_{n=0}^{\infty} \beta_{n} \mathcal{L}\left\{Q_{n}(t)\right\}\right\}, \\
& v_{1}(x)=\mathcal{L}^{-1}\left\{-\frac{1}{s^{n}} \mathcal{L}\left\{H_{0}\left(v_{0}\right)+\sum_{n=0}^{\infty} \beta_{n} Q_{n}(t)-f(t)\right\}\right\}, \\
& v_{2}(x)=\mathcal{L}^{-1}\left\{-\frac{1}{s^{n}} \mathcal{L}\left\{H_{1}\left(v_{0}, v_{1}\right)\right\}\right\}, \\
& v_{3}(x)=\mathcal{L}^{-1}\left\{-\frac{1}{s^{n}} \mathcal{L}\left\{H_{2}\left(v_{0}, v_{1}, v_{2}\right)\right\}\right\}, \\
& \vdots \\
& v_{m}(x)=\mathcal{L}^{-1}\left\{-\frac{1}{s^{n}} \mathcal{L}\left\{H_{m-1}\left(v_{0}, v_{1}, v_{2}, \ldots, v_{m-1}\right)\right\}\right\},
\end{aligned}
$$

Now, let us determine $\beta_{0}, \beta_{1}, \beta_{2}, \ldots$ so that $v_{1}=0$, then from (9) we have $v_{2}=v_{3}=\ldots=0$. Setting $p=1$, results in the solution of equation (3) as the following:

$$
\begin{aligned}
u(t) & =v_{0}(t) \\
& =\mathcal{L}^{-1}\left\{\frac{1}{s} \alpha_{0}+\frac{1}{s^{2}} \alpha_{1}+\cdots+\frac{1}{s^{n}} \alpha_{n-1}+\frac{1}{s^{n}} \sum_{n=0}^{\infty} \beta_{n} \mathcal{L}\left\{Q_{n}(t)\right\}\right\} .
\end{aligned}
$$


Therefore, in this method, only the first He polynomial is calculated, and does not need to solve the differential equation in each iteration. Also we applied just two iterations.

\section{ILLUSTRATIVE EXAMPLES}

In this section, we apply the new method for solution of some ordinary differential equations of Lane-Emden, Ricatti and Euler-Lagrange type.

EXAMPLE 1. In general, the Lane-Emden type equations proposed by Shawagfeh (1993), are formulated as

$u^{\prime \prime}+\frac{\alpha}{t} u+f_{1}(t) g(u)=h(t)$

with initial conditions

$u(0)=A, u^{\prime}(0)=B$.

where $\alpha, A$ and $B$ are real constants and $f_{1}(t), g(y)$ and $h(t)$ are some given functions. For $f_{1}(t)=1, h(t)=0, g(u)=u^{m}, A=1$ and $B=0$, equation (10) is the standard Lane-Emden equation that we used to model the thermal behavior of a spherical cloud of gas acting under the mutual attraction of its molecules and subject to the classical laws of thermodynamics

$u^{\prime \prime}+\frac{2}{t} u+u^{m}=0, t \geq 0$

Subject to the initial conditions

$u(0)=1, u^{\prime}(0)=0$.

where $m \geq 0$ is constant. Substituting $m=0,1$ and $m=5$ into equation (11) leads to the exact solution

$u(t)=1-\frac{t^{2}}{3 !}$,

$u(t)=\frac{\sin (t)}{t}$,

$u(t)=\left(1+\frac{t^{2}}{3}\right)^{-\frac{1}{2}}$

respectively. 
To solve standard Lane-Emden equation (11), by the new method we construct the following equation

$v^{\prime \prime}-\sum_{n=0}^{\infty} \beta_{n} Q_{n}(t)+p\left(\sum_{n=0}^{\infty} \beta_{n} Q_{n}(t)+\frac{2}{t} v^{\prime}+v^{m}\right)=0$

where $L(v)=v^{\prime \prime}, N(v)=\frac{2}{t} v^{\prime}+v^{m}, f=0$ and $H_{0}\left(v_{0}\right)=\frac{2}{t} v_{0}^{\prime}+v_{0}^{m}$.

By applying Laplace transform on both sides of (12), we have

$$
\mathcal{L}\left\{v^{\prime \prime}-\sum_{n=0}^{\infty} \beta_{n} Q_{n}(t)+p\left(\sum_{n=0}^{\infty} \beta_{n} Q_{n}(t)+\frac{2}{t} v^{\prime}+v^{m}\right)\right\}=0
$$

Using the differential property of Laplace transform we have

$$
\begin{aligned}
s^{2} \mathcal{L}\{v\}-s v(0) & -v^{\prime}(0)=\sum_{n=0}^{\infty} \beta_{n} \mathcal{L}\left\{Q_{n}(t)\right\} \\
& -p \mathcal{L}\left\{\sum_{n=0}^{\infty} \beta_{n} Q_{n}(t)+\frac{2}{t} v^{\prime}+v^{m}\right\}
\end{aligned}
$$

or

$$
\begin{aligned}
\mathcal{L}\{v\} & =\frac{1}{s^{2}}\left\{s v(0)+v^{\prime}(0)+\sum_{n=0}^{\infty} \beta_{n} \mathcal{L}\left\{Q_{n}(t)\right\}\right\} \\
& -p \frac{1}{s^{2}} \mathcal{L}\left\{\sum_{n=0}^{\infty} \beta_{n} Q_{n}(t)+\frac{2}{t} v^{\prime}+v^{m}\right\}
\end{aligned}
$$

where $\beta_{0}, \beta_{1}, \beta_{2}, \ldots$ are unknown coefficients, $Q_{n}(t)=t^{n}$ are specific functions depending on the problem, $v(0)=1, v^{\prime}(0)=0$ and $v=\sum_{n=0}^{\infty} p^{n} v_{n}$.

By applying inverse Laplace transform on both sides of (13), we have

$$
\begin{aligned}
\sum_{n=0}^{\infty} p^{n} v_{n} & =\mathcal{L}^{-1}\left\{\frac{1}{s^{2}}\left\{s+\sum_{n=0}^{\infty} \beta_{n} \mathcal{L}\left\{Q_{n}(t)\right\}\right\}\right\} \\
& -p \mathcal{L}^{-1}\left\{\frac{1}{s^{2}} \mathcal{L}\left\{\sum_{n=0}^{\infty} \beta_{n} Q_{n}(t)+\frac{2}{t} v^{\prime}+v^{m}\right\}\right\}
\end{aligned}
$$

According to (9) and (14), we have 


$$
\begin{aligned}
v_{0}(t) & =\mathcal{L}^{-1}\left\{\frac{1}{s}+\frac{1}{s^{2}} \sum_{n=0}^{\infty} \beta_{n} \mathcal{L}\left\{Q_{n}(t)\right\}\right\} \\
& =1+\frac{1}{2} \beta_{0} t^{2}+\frac{1}{6} \beta_{1} t^{3}+\frac{1}{12} \beta_{2} t^{4}+\frac{1}{20} \beta_{3} t^{5}+\cdots \\
v_{1}(t) & =\mathcal{L}^{-1}\left\{-\frac{1}{s^{2}}\left\{\sum_{n=0}^{\infty} \beta_{n} Q_{n}(t)+\frac{2}{t} v_{0}^{\prime}+\frac{2}{t^{2}} v_{0}^{m}\right\}\right\} .
\end{aligned}
$$

Case 1: Assume that $m=0$, if we set $v_{1}(t)=0$ then we have

$$
-\left(\frac{1}{2}-\frac{3}{2} \beta_{0}\right) t^{2}-\frac{1}{3} \beta_{1} t^{3}-\frac{5}{36} \beta_{2} t^{4}-\frac{3}{40} \beta_{3} t^{5}-\frac{7}{150} \beta_{4} t^{6}-\cdots=0 .
$$

This implies that

$$
\beta_{0}=-\frac{1}{3}, \beta_{1}=\beta_{2}=\beta_{3}=\cdots=0 \text {. }
$$

Therefore, the exact solution is recognized easily

$$
u(t)=v_{0}(t)=1-\frac{1}{3 !} t^{2}
$$

Case 2: Assume that $m=1$, if we set $v_{1}(t)=0$ then we have

$$
\begin{aligned}
& -\left(\frac{1}{2}+\frac{3}{2} \beta_{0}\right) t^{2}-\frac{1}{3} \beta_{1} t^{3}-\left(\frac{1}{24} \beta_{0}+\frac{5}{36} \beta_{2}\right) t^{4} \\
& -\left(\frac{1}{120} \beta_{1}+\frac{3}{40} \beta_{3}\right) t^{5}-\left(\frac{1}{360} \beta_{2}+\frac{7}{150} \beta_{4}\right) t^{6}-\cdots=0 .
\end{aligned}
$$

This implies that

$$
\begin{aligned}
& \beta_{0}=-\frac{1}{3}, \beta_{2}=\frac{1}{10}, \beta_{4}=-\frac{1}{168}, \ldots, \\
& \beta_{2 k+1}=0, k=0,1, \ldots .
\end{aligned}
$$

Therefore, the exact solution is recognized easily

$$
\begin{aligned}
u(t)=v_{0}(t) & =1-\frac{1}{6} t^{2}+\frac{1}{120} t^{4}-\frac{1}{5040} t^{6}+\ldots \\
& =1-\frac{1}{3 !} t^{2}+\frac{1}{5 !} t^{4}-\frac{1}{7 !} t^{6}+\ldots \\
& =\frac{\sin (t)}{t} .
\end{aligned}
$$


Case 3: Assume that $m=5$, if we set $v_{1}(t)=0$ then we have

$$
\begin{aligned}
& -\left(\frac{1}{2}+\frac{3}{2} \beta_{0}\right) t^{2}-\frac{1}{3} \beta_{1} t^{3}-\left(\frac{5}{24} \beta_{0}+\frac{5}{36} \beta_{2}\right) t^{4} \\
& -\left(\frac{1}{24} \beta_{1}+\frac{3}{40} \beta_{3}\right) t^{5}-\left(\frac{1}{12} \beta_{0}^{2}+\frac{1}{72} \beta_{2}+\frac{7}{150} \beta_{4}\right) t^{6} \\
& -\left(\frac{5}{126} \beta_{0} \beta_{1}+\frac{1}{168} \beta_{3}+\frac{2}{63} \beta_{5}\right) t^{7}-\cdots=0 .
\end{aligned}
$$

This implies that

$$
\begin{aligned}
& \beta_{0}=-\frac{1}{3}, \beta_{2}=\frac{1}{2}, \beta_{4}=-\frac{75}{216}, \ldots, \\
& \beta_{2 k+1}=0, k=0,1, \ldots
\end{aligned}
$$

Therefore, the exact solution is recognized easily

$$
\begin{aligned}
u(t)=v_{0}(t) & =1-\frac{1}{6} t^{2}+\frac{1}{24} t^{4}-\frac{5}{432} t^{6}+\cdots \\
& =1-\frac{1}{2}\left(\frac{t^{2}}{3}\right)+\frac{\left(\frac{1}{2}\right)\left(\frac{3}{2}\right)}{2 !}\left(\frac{t^{2}}{3}\right)^{2}-\frac{\left(\frac{1}{2}\right)\left(\frac{3}{2}\right)\left(\frac{5}{2}\right)}{3 !}\left(\frac{t^{2}}{3}\right)^{3}+\cdots \\
& =\left(1+\frac{t^{2}}{3}\right)^{-\frac{1}{2}} .
\end{aligned}
$$

EXAMPLE 2. Consider the following Euler-Lagrange equation of order two, with the initial conditions

$$
\begin{aligned}
& u^{\prime \prime}-\frac{2}{t} u^{\prime}+\frac{2}{t^{2}} u=t^{2}+3 t+\frac{1}{t^{2}}, \\
& u(0)=\frac{1}{2}, u^{\prime}(0)=1 .
\end{aligned}
$$

To solve equation (15), by the new method we construct the following equation

$$
v^{\prime \prime}=\sum_{n=0}^{\infty} \beta_{n} Q_{n}(t)-p\left(\sum_{n=0}^{\infty} \beta_{n} Q_{n}(t)-\frac{2}{t} v^{\prime}+\frac{2}{t^{2}} v-t^{2}-3 t-\frac{1}{t^{2}}\right)
$$


where $L(v)=v^{\prime \prime}, N(v)=-\frac{2}{t} v^{\prime}+\frac{2}{t^{2}} v, f=t^{2}+3 t+\frac{1}{t^{2}}$ and

$H_{0}\left(v_{0}\right)=-\frac{2}{t} v_{0}^{\prime}+\frac{2}{t^{2}} v_{0}$.

Using the differential property of Laplace transform we have

$$
\begin{aligned}
& s^{2} \mathcal{L}\{v\}-s v(0)-v^{\prime}(0)=\sum_{n=0}^{\infty} \beta_{n} \mathcal{L}\left\{Q_{n}(t)\right\} \\
& -p \mathcal{L}\left\{\sum_{n=0}^{\infty} \beta_{n} Q_{n}(t)-\frac{2}{t} v^{\prime}+\frac{2}{t^{2}} v-t^{2}-3 t-\frac{1}{t^{2}}\right\}
\end{aligned}
$$

where $\beta_{0}, \beta_{1}, \beta_{2}, \ldots$ are unknown coefficients, $Q_{n}(t)=t^{n}$ are specific functions depending on the problem, $v(0)=\frac{1}{2}, v^{\prime}(0)=1$ and $v=\sum_{n=0}^{\infty} p^{n} v_{n}$.

By applying inverse Laplace transform on both sides of (17), we have

$$
\begin{aligned}
\sum_{n=0}^{\infty} p^{n} v_{n} & =\mathcal{L}^{-1}\left\{\frac{1}{s^{2}}\left\{\frac{1}{2} s+1+\sum_{n=0}^{\infty} \beta_{n} \mathcal{L}\left\{Q_{n}(t)\right\}\right\}\right\} \\
& -p \mathcal{L}^{-1}\left\{\frac{1}{s^{2}} \mathcal{L}\left\{\sum_{n=0}^{\infty} \beta_{n} Q_{n}(t)-\frac{2}{t} v^{\prime}+\frac{2}{t^{2}} v-t^{2}-3 t-\frac{1}{t^{2}}\right\}\right\}
\end{aligned}
$$

According to (18), we have

$$
\begin{aligned}
v_{0}(t) & =\mathcal{L}^{-1}\left\{\frac{1}{2 s}+\frac{1}{s^{2}}+\frac{1}{s^{2}} \sum_{n=0}^{\infty} \beta_{n} \mathcal{L}\left\{Q_{n}(t)\right\}\right\} \\
& =\frac{1}{2}+t+\frac{1}{2} \beta_{0} t^{2}+\frac{1}{6} \beta_{1} t^{3}+\frac{1}{12} \beta_{2} t^{4}+\frac{1}{20} \beta_{3} t^{5}+\cdots, \\
v_{1}(t) & =\mathcal{L}^{-1}\left\{-\frac{1}{s^{2}}\left\{\sum_{n=0}^{\infty} \beta_{n} Q_{n}(t)-\frac{2}{t} v_{0}^{\prime}+\frac{2}{t^{2}} v_{0}-t^{2}-3 t-\frac{1}{t^{2}}\right\}\right\} .
\end{aligned}
$$

Now assume that $v_{1}(t)=0$, then we have

$$
\begin{aligned}
& \left(1-\beta_{0}\right) t+\left(\beta_{0}-\frac{1}{2} \beta_{1}\right) t^{2} \\
& +\left(\frac{1}{3} \beta_{1}-\frac{1}{3} \beta_{0}^{2}-\frac{1}{3} \beta_{2}\right) t^{3}+\left(\frac{1}{6} \beta_{2}-\frac{1}{4} \beta_{0} \beta_{1}-\frac{1}{4} \beta_{3}\right) t^{4} \\
& +\left(\frac{1}{10} \beta_{3}-\frac{1}{20} \beta_{1}^{2}-\frac{2}{15} \beta_{0} \beta_{2}-\frac{1}{5} \beta_{4}\right) t^{5}+\cdots=0 .
\end{aligned}
$$


This implies that

$$
\beta_{1}=9, \beta_{2}=2, \beta_{3}=\beta_{4}=\cdots=0 \text {. }
$$

Therefore, the exact solution is recognized easily

$$
u(t)=v_{0}(t)=\frac{1}{2}+t+\frac{\beta_{0}}{2} t^{2}+\frac{3}{2} t^{3}+\frac{1}{6} t^{4} .
$$

where $\beta_{0}$ is arbitrary.

EXAMPLE 3. Consider the following Riccati equation of order two, with the initial conditions

$$
\begin{aligned}
& u^{\prime}=2 u-u^{2}+1 \\
& u(0)=1 .
\end{aligned}
$$

The exact solution of above equation is

$$
u=1+\sqrt{2} \tanh \left[\sqrt{2} t+\frac{1}{2} \log \left(\frac{\sqrt{2}-1}{\sqrt{2}+1}\right)\right]
$$

The Taylor expansion of $u(t)$ about $t=0$ gives

$$
u(t)=t+t^{2}+\frac{1}{3} t^{3}-\frac{1}{3} t^{4}-\frac{7}{15} t^{5}-\frac{7}{45} t^{6}+\frac{53}{315} t^{7}+\frac{71}{315} t^{8}+\cdots
$$

To solve equation (19), by the new method we construct the following equation

$$
v^{\prime}=\sum_{n=0}^{\infty} \beta_{n} Q_{n}(t)+p\left(\sum_{n=0}^{\infty} \beta_{n} Q_{n}(t)+v^{2}-2 v-1\right)
$$

where $L(v)=v^{\prime}, N(v)=-2 v+v^{2}, f=1$ and $H_{0}\left(v_{0}\right)=v_{0}^{2}-2 v_{0}$.

Using the differential property of Laplace transform we have

$$
s \mathcal{L}\{v\}-v(0)=\sum_{n=0}^{\infty} \beta_{n} \mathcal{L}\left\{Q_{n}(t)\right\}-p \mathcal{L}\left\{\sum_{n=0}^{\infty} \beta_{n} Q_{n}(t)+v^{2}-2 v-1\right\}
$$

where $\beta_{0}, \beta_{1}, \beta_{2}, \ldots$ are unknown coefficients, $Q_{n}(t)=t^{n}$ are specific functions depending on the problem, $v(0)=1$ and $v=\sum_{n=0}^{\infty} p^{n} v_{n}$.

By applying inverse Laplace transform on both sides of (22), we have 


$$
\begin{aligned}
\sum_{n=0}^{\infty} p^{n} v_{n} & =\mathcal{L}^{-1}\left\{\frac{1}{s}\left\{1+\sum_{n=0}^{\infty} \beta_{n} \mathcal{L}\left\{Q_{n}(t)\right\}\right\}\right\} \\
& -p \mathcal{L}^{-1}\left\{\frac{1}{s} \mathcal{L}\left\{\sum_{n=0}^{\infty} \beta_{n} Q_{n}(t)+v^{2}-2 v-1\right\}\right\}
\end{aligned}
$$

According to (23), we have

$$
\begin{aligned}
v_{0}(t) & =\mathcal{L}^{-1}\left\{\frac{1}{s}+\frac{1}{s} \sum_{n=0}^{\infty} \beta_{n} \mathcal{L}\left\{Q_{n}(t)\right\}\right\} \\
& =\beta_{0} t+\frac{1}{2} \beta_{1} t^{2}+\frac{1}{3} \beta_{2} t^{3}+\frac{1}{4} \beta_{3} t^{4}+\cdots, \\
v_{1}(t) & =\mathcal{L}^{-1}\left\{-\frac{1}{s}\left\{\sum_{n=0}^{\infty} \beta_{n} Q_{n}(t)+v_{0}^{2}-2 v_{0}-1\right\}\right\} .
\end{aligned}
$$

Now assume that $v_{1}(t)=0$, then we have

$$
\begin{aligned}
& \left(\frac{1}{2}-\frac{1}{18} \beta_{1}\right) t^{3}+\left(\frac{1}{12}-\frac{1}{24} \beta_{2}\right) t^{4} \\
& -\frac{3}{100} \beta_{3} t^{5}-\frac{1}{45} \beta_{4} t^{6}-\frac{5}{294} \beta_{5} t^{7}-\frac{3}{224} \beta_{5} t^{8}-\frac{7}{648} \beta_{7} t^{9}-\cdots=0,
\end{aligned}
$$

equating the coefficients of $t^{m}, m=3,4, \ldots$ to zero, then we have

$$
\beta_{0}=1, \beta_{1}=2, \beta_{2}=1, \beta_{3}=-\frac{4}{3}, \beta_{4}=-\frac{7}{3}, \beta_{5}=-\frac{14}{15}, \cdots .
$$

Therefore, the solution of the Riccati equation will be obtained as follows

$$
\begin{gathered}
u(t)=v_{0}(t)=t+t^{2}+\frac{1}{3} t^{3}-\frac{1}{3} t^{4}-\frac{7}{15} t^{5}-\frac{7}{45} t^{6}+\frac{53}{315} t^{7}+\cdots \\
=1+\sqrt{2} \tanh \left[\sqrt{2} t+\frac{1}{2} \log \left(\frac{\sqrt{2}-1}{\sqrt{2}+1}\right)\right]
\end{gathered}
$$

which is an exact solution. 


\section{CONCLUSION}

In this paper, we proposed the new algorithm to solve ODEs. The numerical solutions are compared with the exact solutions in three examples. The results show that this iterative scheme provides excellent approximations to the solution of this nonlinear equation with high accuracy by applying just two iterations.

\section{REFERENCES}

NAYFEH, A. H. 2011. Introduction to perturbation techniques, Wiley-VCH.

RAND, R. H., ARMBRUSTER, D. AND RAND, R. 1987. Perturbation methods, bifurcation theory and computer algebra, Springer-Verlag, New York.

HE, J. H. 1999. Homotopy perturbation technique. Computer Methods in Applied Mechanics and Engineering $178,257-262$.

GANJI, D. D. 2006. The application of He's homotopy perturbation method to nonlinear equations arising in heat transfer. Physics Letters A 355, 337-341.

ABBASBANDY, S. 2006. Numerical solutions of the integral equations: Homotopy perturbation method and Adomian's decomposition method. Applied Mathematics and Computation 173, 493-500.

YILDIRIM, A. AND KOCAK, H. 2009. Homotopy perturbation method for solving the space-time fractional advection-dispersion equation. Advances in Water Resources 32, 1711-1716.

SHAKERI, F. AND DEHGHAN, M. 2008. Solution of delay differential equations via a homotopy perturbation method. Mathematical and Computer Modelling 48, 486-498.

AMINIKHAH, H. 2010. An analytical approximation for solving nonlinear Blasius equation by NHPM. Numerical Methods for Partial Differential Equations 26, 1291-1299.

AMINIKHAH, H. AND BIAZAR, J. 2009. A new HPM for ordinary differential equations. Numerical Methods for Partial Differential Equations 26, 480-489.

BILDIK, N., KONURALP, A., ORAKCL, B. AND KUCUKARSLAN, S. 2006. Solution of different type of the partial differential equation by differential transform method and Adomian's decomposition method. Applied Mathematics and Computation 172, 551-567.

BIAZAR, J., BABOLIAN, E. AND ISLAM, R. 2004. Solution of the system of ordinary differential equations by Adomian decomposition method. Applied Mathematics and Computation 147, 713-719.

BABOLIAN, E., Biazar, J. AND Vahidi, A.R. 2004. Solution of a system of nonlinear equations by Adomian decomposition method. Applied Mathematics and Computation 150, 847-854.

PAMUK, S. 2005. Solution of the porous media equation by Adomian's decomposition method. Physics Letters A $344,184-188$.

LIAO, S. 2004. On the homotopy analysis method for nonlinear problems. Applied Mathematics and Computation 147, 499-513.

ABBASBANDY, S. 2006. The application of homotopy analysis method to nonlinear equations arising in heat transfer. Physics Letters A 360, 109-113.

JAFARI, H. AND SEIFI, S. 2009. Homotopy analysis method for solving linear and nonlinear fractional diffusion-wave equation. Communications in Nonlinear Science and Numerical Simulation 14, 2006-2012.

ODIBAT, Z., MOMANI, S. AND ERTURK, S. 2008. Generalized differential transform method: Application to differential equations of fractional order. Applied Mathematics and Computation 197, 467-477.

ERTURK, S. AND MOMANI, S. 2008. Solving systems of fractional differential equations using differential transform method. Journal of Computational and Applied Mathematics 215, 142-151.

AYAZ, F. 2004. Solutions of the system of differential equations by differential transform method. Applied Mathematics and Computation 147, 547-567.

GHORBANI, A. 2009. Beyond Adomian polynomials: He polynomials. Chaos, Solitons \& Fractals 39, 14861492.

SHAWAGFEH, N.T. 1993. Nonperturbative approximate solution for Lane-Emden equation. J. Math. Phys. 34, 4364-4369. 
Hossein Aminikhah

Department of Applied Mathematics

School of Mathematical Sciences

University of Guilan

P.O. Box 41335-1914, Rasht, Iran

Received May 2012 